\title{
Pragmatic research tales: The ELEVATE study as an illustration of the issues involved in implementing pragmatic research
}

\section{Annie Blyth, Stan Musgrave, David Price, Ian Harvey}

\author{
Background: \\ Despite increased asthma preventative treatment there is evidence \\ that large numbers of patients continue to suffer significant \\ symptoms and lifestyle limitation. Possible reasons include lack of \\ disease recognition, poor adherence with inhaled corticosteroids and \\ inability of inhaled steroids to fully control asthma. \\ As earlier studies have been short term, and far from "real-life", the \\ NHS' National Coordinating Centre for Health Technology \\ Assessment sought a study to look at both the short and long term \\ impact on quality of life, clinical status and resource utilisation of \\ using leukotriene receptor antagonists (LTRAs). ELEVATE was
}

conceived in response to this need and the GPIAG was successful in winning this research bid in conjunction with the University of East Anglia.

Study objectives and design:

The study's objective is to compare quality of life comparing LTRAs with alternative therapies at steps 2 \& 3 of the National Asthma Guidelines for 720 adult patients. The study is a pragmatic randomised controlled trial over 2 years that will provide information about broadly generalisable patients in "real world" general practice.

\section{Annie Blyth}

University of Aberdeen

\section{Stan Musgrave}

University of Aberdeen

\section{David Price}

University of Aberdeen

\section{Ian Harvey}

University of Aberdeen

Correspondence to:

Annie Blyth

Dept of General Practice \& Primary Care University of Aberdeen Westburn Road Aberdeen

Tel: + $44(0) 1224552427$ Fax: + $44(0) 1224550683$

Date submitted: 04/08/03 Date Accepted: 02/09/03

Prim Care Resp J 2003; 12(3):95

\section{Pragmatic studies:}

A pragmatic study assesses the effect of an intervention in circumstances that are as close as possible to normal routine care for both patient and practice. For both, there should be no incentive or activity that would alter compliance, and care must be maintained as normal. Consequently, practices need extensive and highly customised support which makes participating in the study easier, but does not bias or substantially alter a patients' normal expected standard of care.

Challenges of pragmatic studies: At the patient level this means accommodating patients' normal behaviour and their unique and often varying need for therapy, thus reflecting real life more accurately than more classical RCTs. Practices, like patients, differ considerably, consequently a pragmatic study must have flexible study processes and implementation. Researchers typically expect to deal with issues of eligibility, randomisation, ethics and research governance, etc., but aren't necessarily prepared to adapt to the inevitable diversity of practice systems and personnel. We found that the specific support we needed to give to practices evolved with the study, engendering a symbiotic and mutually beneficial relationship. Given the individualised implementation at each practice, adaptations were in response to practice requests and under their control and always aimed to use practice time effectively, so clinicians' time is spent with patients, not study procedures.

Examples of problems and solutions found were:

- Nursing time - a group of study nurses were enlisted to assist with the study, being accompanied by a researcher to ensure minimum disruption to the practice

- Telephoning eligible patients to arrange initial appointments - staff were provided to help with this for all centres; phoning patients in the evenings and Saturday mornings.

- Patients not meeting entry criteria when seen phone calls are structured to confirm patients are symptomatic and/or have impaired quality of life and thus require an increase in treatment.

- DNAs - the study office rings patients to remind them of imminent follow-up appointments

\section{Recruiting GPs}

It is becoming increasingly difficult to recruit practices into research projects, due to increasing demands and pressures on them and their practice resources. To be effective in enrolling GPs we had to convince them that we really could guarantee substantial and relevant help, although many simply couldn't accept this as prior experience had suggested the opposite. However, we are successfully confronting and overcoming such challenges in over forty practices in Norfolk, Suffolk, Essex and Bedfordshire.

\section{Identifying and recruiting patients:}

Identifying and recruiting possible participants, particularly within a well-researched population, is a universal problem. Experience shows that relying on clinicians to do this is likely to lead to a biased sample. Consequently the research team assumed this responsibility, by performing searches of computerised records to identify those receiving asthma treatment followed up with postal questionnaires to identify those with impaired asthma control or quality of life.

Over 37 practices and 350 patients have been recruited to participate so far. Some initial results are in the GPIAG ASM abstract by Murdoch. ${ }^{1}$ While we started in East Anglia, we are now moving more widely to other regions of the country, so anyone interested in participating, please contact us at elevate@uea.ac.uk or 01603-591106.

Thus we have learned that implementing a study of this type requires:

- a substantial change in mind-set to adapt to a very different conception of "what an RCT is",

- a commitment to listening to individual practices and overcoming each obstacle,

- a great deal of customised support to enable practices and patients to participate and remain active in the process,

- enthusiasm and perseverance.

\section{References}

1 Murdoch, J. et al., Profiles of Asthma control in 12488 patients in 30 practices in East Anglia. PCRJ 2003;12(2):70 